\title{
L'écriture du scepticisme chez Montaigne, «Actes des journées d'étude (15-16 novembre 2001)», réunis et publiés par Marie-Luce DEMONET et Alain LEGROS
}

\section{Sabine Lardon}

\section{(C) OpenEdition}

1 Journals

\section{Édition électronique}

URL : https://journals.openedition.org/studifrancesi/26137

DOI : 10.4000/studifrancesi.26137

ISSN : 2421-5856

Éditeur

Rosenberg \& Sellier

\section{Édition imprimée}

Date de publication : 1 avril 2007

Pagination : 164-165

ISSN : 0039-2944

\section{Référence électronique}

Sabine Lardon, «L'écriture du scepticisme chez Montaigne, «Actes des journées d'étude (15-16

novembre 2001)», réunis et publiés par Marie-Luce DEMONET et Alain LEGros », Studi Francesi [En ligne], 151 (LI | I) | 2007, mis en ligne le 30 novembre 2015, consulté le 23 novembre 2021. URL : http:// journals.openedition.org/studifrancesi/26137; DOI : https://doi.org/10.4000/studifrancesi.26137

Ce document a été généré automatiquement le 23 novembre 2021.

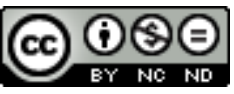

Studi Francesi è distribuita con Licenza Creative Commons Attribuzione - Non commerciale - Non opere derivate 4.0 Internazionale. 


\title{
L'écriture du scepticisme chez
} Montaigne, «Actes des journées d'étude (15-16 novembre 2001)», réunis et publiés par Marie-Luce DEMONET et Alain LEGROS

\author{
Sabine Lardon
}

\section{RÉFÉRENCE}

L'écriture du scepticisme chez Montaigne, «Actes des journées d'étude (15-16 novembre 2001)», réunis et publiés par Marie-Luce DEMONET et Alain LEGROS, Genève, Droz, 2004, pp. 347.

Dans son introduction, («Entrée»: «Que sais-je de Montaigne?»), A. LEGROs rappelle les principes de ce colloque pour lequel littéraires et philosophes ont réfléchi de concert au travail sceptique de Montaigne à partir des trois discours scientifiques et universitaires de l'époque: théologie, droit et médecine.

2 La première partie, «Traits», s'intéresse à l'écriture du scepticisme dans les Essais. Les deux premières interventions concernent l'écriture et la démarche sceptique dans sa généralité: J. O’BRIEN ( «Si avons nous une tres-douce medecine que la philosophie», pp. 13-24) étudie la rhétorique purgative de Montaigne à partir du parallèle tiré par Sextus Empiricus entre la démarche du philosophe sceptique et celle du médecin, tandis que $\mathrm{K}$. SELLEVOLD («Phônai skeptikai et expressions modalisantes. Ressemblances et différences», pp. 25-37) évalue ressemblances et différences entre expressions sceptiques (pas plus, peut-être, admettons, je ne définis rien, je suspends mon jugement) et expressions modalisantes (peut-être, à l'aventure, il me semble, je pense, je trouve). Les trois interventions suivantes se concentrent sur l'«Apologie» et la question de la foi en 
particulier. A. LEGROS («Colloque pour voix sceptiques et parole(s) divine(s) entre librairie et "Apologie"», pp. 39-62) nous invite à lire «l'Apologie de Raimond Sebond» (II.12) comme un texte où Montaigne se révèle véritablement, par-delà l'œuvre de commande, comme en témoignent les sentences bibliques ou sceptiques communes au chapitre et aux poutres de sa «librairie». s. GIOCANTI («Quelle place pour Dieu au sein du discours sceptique de Montaigne?», pp. 63-76) remarque que les occurrences du mot Dieu (et dérivés) dans les Essais (dont plus d'un tiers en II.12) relèvent d'une tradition culturelle de par leur lexicalisation et ne contredisent donc pas un scepticisme opposé à toute démarche théologique. M. HABERT («Aspects sceptiques de la traduction de Sebond», pp. 77-106) montre comment, par-delà une apparente fidélité, la traduction de Montaigne de la Scientia Libri Creaturarum de Raimond Sebond commandée par son père fait appraître de significatifs écarts de détail, témoins d'une distanciation sceptique du traducteur.

3 La deuxième partie, «Conférences», compare les Essais à d'autres écrits de l'époque. J.-c. MARGOLIN («D'Erasme à Montaigne: l'écriture de l'opinion et la double voie de la croyance», pp. 109-129) rapproche les démarches intellectuelles d'Erasme et de Montaigne autour des deux notions-clés d'opinion et de croyance, tout en distinguant leurs spécificités. B. PINCHARD («Humanisme de la chose, humanisme de la glose. Cajétan et Montaigne», pp. 131-142) se fait l'écho des propos de J. Céard qui invitait, en 1981, à considérer les Essais comme «une libre efflorescence du genre du commentaire». En prenant pour point de comparaison l'œuvre de Cajétan, grand commentateur thomiste du début $\mathrm{du} \mathrm{xvI}^{\mathrm{e}}$ siècle, il montre en quoi le texte montaigniste s'avère «la transgression volontaire d'une ontologie déterminée». C'est au modèle juridique que les interventions suivantes confrontent les Essais: S. GEONGET («Perplexité et scepticisme dans les Essais ou la souris et le ver à soie. [III, I]», pp. 143-157) montre comment la perplexité prend, chez Montaigne, le contrepied de la démarche juridique et théologique pour étudier l'image symbolique du ver à soie (III.1). O. GUERRIER («Le Champ du "possible": de la jurisprudence aux Essais», pp. 159-168) étudie, à partir de sa définition juridique, la notion de "possible» dans les Essais. K. ALMQUIST («Du prêt et de l'usufruit des images. Le droit de la propriété dans la pensée sceptique de Montaigne», pp. 169-177) s'intéresse à trois juristes dont la pensée présente, comme chez Montaigne, un syncrétisme stoïco-sceptique pour montrer ensuite comment l'Apologie (II.12) suggère «un modèle de la notion d'une image basée sur le droit de l'usufruit» (p. 176). P. DESAN («Montaigne et le doute judiciaire», pp. 179-187) rappelle à juste titre que le scepticisme de Montaigne ne saurait supposer l'adhésion à un dogme chez celui qui renvendique ne pas être philosophe et montre comment le doute montaigniste se distingue du doute judiciaire.

4 La troisième partie, «Dogmes», confronte le doute sceptique et la foi chez Montaigne. J.L. VIEILLARD-BARON («Croire ou ne pas croire? Montaigne et la foi», pp. 191-199) souligne la position centrale de la foi chez Montaigne. E. NAYA («Le doute libérateur: préambules à une étude du discours fidéiste dans les Essais», pp. 201-221) dénonce l'anachronisme des critiques qui appliquent à Montaigne la notion de fidéisme pour repenser le rapport entre épistémologie pyrrhonienne et acte de foi catholique dans les Essais. T. GONTIER («L'essai et l'expérience. Le scepticisme montaigniste par-delà le fidéisme», pp. 223-237) se propose d'évaluer le sens et les enjeux du scepticisme montaigniste dont il dégage, en particulier, une définition de l'expérience. N. PANICHI («La raison sceptique comme figure de l'éthique», pp. 239-261) met en tension le néo-pyrrhonisme de 
Montaigne et sa recherche d'une vérité à la mesure de l'homme pour étudier les enjeux d'un discours montaigniste fondé sur la conversation-communication.

5 La quatrième partie, «Expériences», appuie l'étude du scepticisme de Montaigne sur des éléments biographiques. A. TOURNON («La question du Préteur», p. 265-274) part de la question de Pilate rapportée par saint Jean (XVIII.37-38 - interrogatoire de Jésus): «Qu'est-ce que la vérité?» pour rappeler la procédure juridique qu'a pu pratiquer Montaigne en tant que conseiller de la Chambre des Enquêtes et dont son pyrrhonisme pourrait être tributaire. J. BALSAMO («La critique des dispositions testamentaires: un scepticisme peu philosophique», pp. 275-287) donne une étude philologique et stylistique du chapitre II.8 en montrant que les formules d'apparence sceptique concernant la question des dispositions testamentaires contribuent, en fait, à autocélébrer la grandeur d'âme de l'auteur. G. DEFAUX («Montaigne et l'expérience: réflexions sur la naissance d'un philosophe sceptique - et "impremedité"», pp. 289-302) invite à rejeter «une bonne foi pour toutes» la fameuse thèse «évolutive» de P. Villey et à situer la genèse du livre non pas dans sa librairie, mais dans la chambre de l'ami agonisant (La Boétie). D. BRANCHER («Ny plus ne moins que la rubarbe qui pousse hors les mauvaises humeurs: la rhubarbe au Purgatoire», pp. 303-320) renoue avec le sujet de la première intervention pour confronter la démarche des Essais à celle de Sextus Empiricus. En conclusion («Sortie»: «Du jeton à l'éponge», p. 321), M.-L. DEMONET met en relation le fameux jeton de Montaigne avec le texte sceptique des Essais.

Une bibliographie et un index des noms de personne complètent ces Actes qui font montre d'une belle cohérence dans le propos. S'en dégagent d'une part la figure d'un Montaigne unique par sa pensée et sa démarche, se démarquant des modèles de son temps (démarche juridique, commentaire théologique, etc.) par-delà même ses points de contact; d'autre part l'image d'une pensée sceptique affranchie de toute soumission à un dogme philosophique. Amusant constat, cette dérobade du libre-penseur se retrouve jusque dans la difficulté à le qualifier: montaigniste, montaniste ou montaignien, les chercheurs ici réunis hésitent - un doute qui aurait été loin de déplaire à Montaigne... 